\title{
Transient Solution for the Energy Balance in Porous Media Considering Viscous Dissipation and Expansion/Compression Effects Using Integral Transforms
}

\author{
Ricardo Hüntemann Deucher ${ }^{1} \cdot$ Paulo Couto $^{2}$ (D) \\ Gustavo César Rachid Bodstein ${ }^{3}$
}

Received: 25 May 2016 / Accepted: 18 November 2016/ Published online: 1 December 2016

(C) Springer Science+Business Media Dordrecht 2016

\begin{abstract}
Monitoring of downhole flowing temperatures in oil wells is gaining attention in the recent years due to the possibility of exploring these data for reservoir characterization and determination of inflow profiles along the well completions, leading to an increased interest in the development of solutions for the equations governing the thermal behavior of a reservoir. In this work, it is proposed to use the generalized integral transform technique (GITT) to provide solutions for the energy balance equation, considering the thermal effects related to fluid flow. A formal and general solution for the energy balance in the porous media is presented and validated. It is presented the application of the proposed solution to one-dimensional and two-dimensional problems in the Cartesian coordinate system. The two-dimensional problem, which considers heat transfer to the surrounding impermeable formations, is tackled by a single domain formulation. The mathematical approach taken in these solutions is rigorous, valid for all flow regimes (transient, late-transient and pseudo-steadystate/steady-state) and for any orthogonal coordinate system, presenting the possibility of achieving differentiable and stable solutions with controlled accuracy. The solution comprises an important contribution to support the application of temperature data to reservoir engineering problems.
\end{abstract}

Keywords Energy balance $\cdot$ Temperature transient $\cdot$ Integral transform $\cdot$ Porous media

$\triangle$ Ricardo Hüntemann Deucher rhdeucher@gmail.com

1 Petrobras S/A, Av. Republica do Chile 330, Centro, Rio de Janeiro, Brazil

2 Program of Civil Engineering, COPPE, Federal University of Rio de Janeiro, UFRJ, Rio de Janeiro, Brazil

3 Program of Mechanical Engineering, COPPE, Federal University of Rio de Janeiro, UFRJ, Rio de Janeiro, Brazil 


\section{List of Symbols}

\begin{tabular}{ll}
$A_{i j}^{*}$ & Expansion coefficients \\
$a(\boldsymbol{x}), b(\boldsymbol{x})$ & Boundary condition coefficients (temperature) \\
$c(\boldsymbol{x}), d(\boldsymbol{x})$ & Boundary condition coefficients (pressure) \\
$\overline{C_{p}}$ & Average-specific heat capacity of rock/fluid \\
$C_{p r}$ & Rock-specific heat capacity \\
$C_{p f}$ & Fluid-specific heat capacity \\
$c_{t}$ & Total compressibility \\
$c_{r}$ & Rock compressibility \\
$\bar{f}$ & Transformed initial condition (temperature) \\
$f(\boldsymbol{x})$ & Initial condition (temperature) \\
$\bar{g}$ & Transformed source term \\
$k$ & Permeability \\
$k_{T}$ & Thermal conductivity \\
$L_{x}, L_{y}, L_{z}$ & Reservoir dimensions \\
$N$ & Normalization integrals \\
$\boldsymbol{n}$ & Normal vector \\
$p$ & Pressure \\
$Q$ & Flowrate \\
$S$ & Domain surface \\
$T$ & Temperature \\
$\bar{T}$ & Transformed potential \\
$T_{0}$ & Initial undisturbed reservoir temperature \\
$T_{1}$ & Parameter of Eq. (10) \\
$t$ & Time \\
$V$ & Domain region \\
$\boldsymbol{v}$ & Velocity vector \\
$W_{p}$ & Source term \\
$x_{c}$ & Parameter of Eq. (10) \\
$x_{p}$ & Well position \\
$\boldsymbol{x}$ & Position vector \\
& \\
\hline &
\end{tabular}

\section{Greek Symbols}

$\begin{array}{ll}\beta & \text { Thermal expansion coefficient } \\ \eta & \text { Hydraulic diffusivity constant } \\ \mu & \text { Viscosity } \\ \mu_{J T} & \text { Joule-Thomson coefficient } \\ \Upsilon(\boldsymbol{x}) & \text { Initial condition (pressure) } \\ \phi & \text { Porosity } \\ \bar{\rho} & \text { Average density of rock/fluid } \\ \rho_{r} & \text { Rock density } \\ \rho_{f} & \text { Fluid density } \\ \xi(\boldsymbol{x}, t) & \text { Boundary condition coefficients (pressure) } \\ \varphi(\boldsymbol{x}, t) & \text { Boundary condition coefficients (temperature) } \\ \psi_{i}(x), \quad \Omega_{j}(z) & \text { Eigenfunctions } \\ \sigma_{i}, \chi_{j} & \text { Eigenvalues } \\ \omega & \text { Parameter of Eq. (10) }\end{array}$




\section{Introduction}

Due to improvements on precision and reliability of tools that measure temperature in oil wells, there is an increasing interest in the development of mathematical models to interpret downhole temperature data, aiming to use them as a complimentary source of information to pressure data, which are traditionally used for formation evaluation purposes.

The development of solutions for the energy balance in porous media considering viscous dissipation and expansion/compression effects is fundamental to the use of temperature data for reservoir characterization. In the last years, several authors presented solutions for the energy balance in porous media. In an early effort, Maubeuge et al. (1994), aiming to use temperature data for production logging interpretation, presented a numerical solution for the transient energy balance considering the Joule-Thomson effect in the model. Yoshioka et al. (2005), aiming to investigate the effects of water or gas entry into a horizontal well, presented an analytical solution for the energy balance in porous media considering steady-state fluid flow. Ramanazov and Nagimov (2007) presented an analytical solution that considers transient radial flow in a reservoir with infinite hydraulic diffusivity; their model neglects the heat transfer to the adjacent impermeable formations. Duru (2011) developed a numerical, as well as a semi-analytical solution for the transient energy balance using operator splitting method, presenting applications of downhole temperature data to improve the estimation of reservoir porosity and permeability fields. Muradov and Davies (2011) developed an asymptotic analytical solution for the transient two-dimensional energy balance; their solution is able to represent the thermal behavior in horizontal wells, considering linear fluid flow and the heat transfer with the surrounding formations. Despite these advances, there is still a need for the development of generalized, exact and stable solutions for the transient energy balance considering viscous dissipation and expansion/compression effects.

The classical integral transform technique (CITT) is a method for the solution of linear diffusion problems based on the method of separation of variables, which expands the solution in terms of a series of solutions to auxiliary eigenvalue problems. The use of the CITT to obtain analytical solutions of seven classes of linear diffusion problems has been presented and analyzed by Mikhailov and Ozisik (1984).

Several authors have presented applications of the CITT to solve the problem of fluid flow in porous media. Hovanessian (1961) and Rahman and Bentsen (2000) presented applications of the CITT to solve the hydraulic diffusivity equation (HDE) in the Cartesian coordinate system. Later, Rahman and Bentsen (2001) extended their previous work to solve the HDE for problems in the radial coordinate system.

An extension of the analytical ideas behind the CITT led to the development of the generalized integral transform technique (GITT) (Cotta, integral transforms in computational heat and fluid flow, 1993), which offers more flexibility in handling a priori non-transformable problems and allows the solution of more complex situations, such as the solution of nonlinear diffusion and convection-diffusion problems (Cotta, Benchmark results in computational heat and fluid flow: the integral transform method, 1994). The work of Almeida and Cotta (1995) presented the application of the GITT to solve the tracer injection problem in petroleum reservoirs.

The aim of this work is to develop a generalized and complete hybrid solution for the energy balance in porous media utilizing the GITT. This solution considers thermal effects caused by fluid flow (expansion/compression and viscous dissipation), rock expansion/compression, heat conduction and advection. Due to its analytical nature, the hybrid GITT solution pre- 
sented retains important features of analytical solutions that differentiate them from purely numerical schemes: (a) stability; (b) controlled accuracy; (c) can be used to provide trends and asymptotic behaviors, without requiring a complete numerical solution; (d) can provide benchmark results for the validation of numerical schemes applicable to more involved situations; and (e) are more amenable to mathematical treatments for inverse problems and parameters estimation.

\section{Problem Formulation}

Figure 1 presents an illustrative scheme of the problem to be solved in this work, describing the heat transfer mechanisms as well as the source terms affecting the transient thermal behavior. Fig. 1 represents an advection-diffusion problem with source terms related to viscous dissipation and expansion/compression of rock and fluid. These source terms are related to changes in pressure that occur inside the reservoir, in such a way that, to solve the energy balance, it is necessary to solve the hydraulic diffusivity equation, which describes the transient and spatial pressure variations that take place inside a producing reservoir. The HDE neglecting gravitational effects and considering the flow of liquids of low and constant compressibility with a source term representing the well production $\left(W_{p}\right)$ can be expressed by the following formulation (Deucher et al. 2016):

$$
\phi(\boldsymbol{x}) c_{t} \frac{\partial p(\boldsymbol{x}, t)}{\partial t}=\nabla \cdot\left(\frac{k(\boldsymbol{x})}{\mu} \nabla p(\boldsymbol{x}, t)\right)+W_{p}(\boldsymbol{x}, t), \boldsymbol{x} \in V, t>0
$$

with generalized boundary conditions:

$$
c(\boldsymbol{x}) p(\boldsymbol{x}, t)+d(\boldsymbol{x}) \frac{k(\boldsymbol{x})}{\mu} \frac{\partial p(\boldsymbol{x}, t)}{\partial \boldsymbol{n}}=\xi(\boldsymbol{x}, t), \boldsymbol{x} \in S, t>0
$$

and generalized initial condition:

$$
p(\boldsymbol{x}, t)=\Upsilon(\boldsymbol{x}), \boldsymbol{x} \in V, t=0
$$

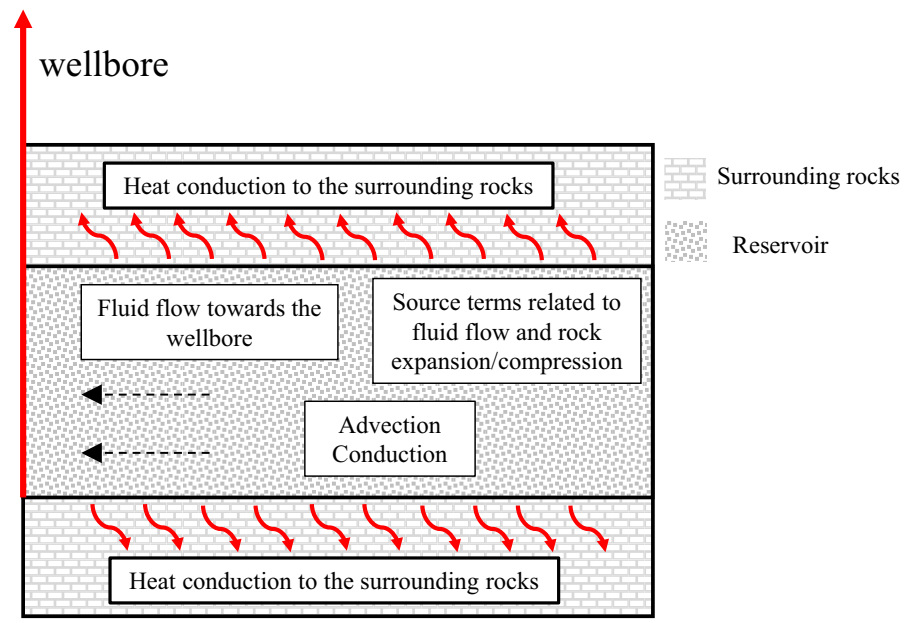

Fig. 1 Illustrative scheme of the problem to be solved in this work 
where $c_{t}$ represents the total compressibility of the reservoir, accounting for the rock $\left(c_{r}\right)$ and fluids present at the pore space compressibilities (oil and water).

Deucher et al. (2016) presented a generalized solution for problem (1) that is going to be considered throughout the applications presented in this work. A detailed explanation and demonstration of this solution, which is based on eigenfunction expansions, can be found in Deucher et al. (2016). It is important to mention that the solution of the energy balance that is presented does not require the use of the generalized solution of the HDE presented by Deucher et al. (2016), being it possible to use any other solutions of the HDE found in the literature, such as the ones presented by Carslaw and Jaeger (1959).

The energy balance, as derived by Sui et al. (2008), is given by:

$$
\begin{aligned}
\bar{\rho} \bar{C}_{p} & \frac{\partial T(\boldsymbol{x}, t)}{\partial t}+\rho_{f} C_{p f} \boldsymbol{v}(\boldsymbol{x}, t) \cdot \nabla T(\boldsymbol{x}, t) \\
= & \phi(\boldsymbol{x}) \beta T(\boldsymbol{x}, t) \frac{\partial p(\boldsymbol{x}, t)}{\partial t}+\left(p(\boldsymbol{x}, t)+\rho_{r} C_{p r} T(\boldsymbol{x}, t)\right) \phi c_{r} \frac{\partial p(\boldsymbol{x}, t)}{\partial t} \\
& +(\beta T(\boldsymbol{x}, t)-1) \boldsymbol{v}(\boldsymbol{x}, t) \cdot \nabla p(\boldsymbol{x}, t)+\nabla \cdot\left(k_{T}(\boldsymbol{x}) \nabla T(\boldsymbol{x}, t)\right), \\
& \quad \boldsymbol{x} \in V, \quad t>0 .
\end{aligned}
$$

As observed by Muradov and Davies (2011), in transient temperature analysis, the temperature variations are generally small $(\sim 2 \mathrm{~K})$ when compared to the initial reservoir temperature $\left(T_{0} \sim 350 \mathrm{~K}\right)$, making it possible to consider, without significant loss of accuracy, that $\phi(\boldsymbol{x}) \beta T(\boldsymbol{x}, t) \cong \phi(\boldsymbol{x}) \beta T_{0}, \rho_{r} C_{p r} T(\boldsymbol{x}, t) \cong \rho_{r} C_{p r} T_{0}$ and $(\beta T(\boldsymbol{x}, t)-1) \cong\left(\beta T_{0}-1\right)$, allowing one to linearize Eq. 2:

$$
\begin{aligned}
\bar{\rho} & \overline{C_{p}} \\
= & \phi(\boldsymbol{x}) \beta T_{0} \frac{\partial p(\boldsymbol{x}, t)}{\partial t}+\rho_{f} C_{p f} \boldsymbol{v}(\boldsymbol{x}, t) \cdot \nabla T(\boldsymbol{x}, t) \\
& \left.+\left(\beta T_{0}-1\right) \boldsymbol{v}(\boldsymbol{x}, t) . \nabla p(\boldsymbol{x}, t)+\rho_{r} C_{p r} T_{0}\right) \phi c_{r} \frac{\partial p(\boldsymbol{x}, t)}{\partial t}+\nabla \cdot\left(k_{T}(\boldsymbol{x}) \nabla T(\boldsymbol{x}, t)\right), \\
& \boldsymbol{x} \in V, \quad t>0
\end{aligned}
$$

with generalized boundary conditions:

$$
a(\boldsymbol{x}) T(\boldsymbol{x}, t)+b(\boldsymbol{x}) k_{T}(\boldsymbol{x}) \frac{\partial T(\boldsymbol{x}, t)}{\partial \boldsymbol{n}}=\varphi(\boldsymbol{x}, t), \boldsymbol{x} \in S, \quad t>0
$$

and generalized initial condition:

$$
T(\boldsymbol{x}, t)=f(\boldsymbol{x}), \boldsymbol{x} \in V, \quad t=0
$$

the velocity, $\boldsymbol{v}(\boldsymbol{x}, t)$, is given by Darcy's Law:

$$
\boldsymbol{v}(\boldsymbol{x}, t)=-\frac{k(\boldsymbol{x})}{\mu} \nabla p(\boldsymbol{x}, t)
$$

Equation (3a) states that the variations of temperature (1st term on the left-hand side) are ruled by a series of energy transport and heat release phenomena:

(a) advection (2nd term on the left-hand side);

(b) transient expansion/compression of the fluid (1st term on the right-hand side);

(c) transient expansion/compression of the rock (2nd term on the right-hand side);

(d) spatial expansion/compression of the fluid and viscous dissipation (3rd term on the righthand side). This term, in spite of being directly related to the Joule-Thomson coefficient 
of the fluid, which is given by $\mu_{J T}=\left(\beta T_{0}-1\right) / \rho_{f} C_{p f}$, is not the manifestation of the Joule-Thomson effect itself;

(e) heat conduction (4th term on the right-hand side).

\section{Formal Solution}

The solution of the energy balance given by Eq. (3a-c) can be obtained by the GITT. According to Cotta (1993), the basic steps involved in the application of the GITT are: (a) choice of an appropriate eigenvalue problem; (b) development of the integral transform pair; (c) attempt to integral transform the original partial differential equation; (d) truncate the infinite system of coupled ordinary differential equation and solve it to obtain the transformed potentials; and (e) use the inverse formula to obtain the original potential.

The basic steps presented above are applied to the problem given by Eq. (3a-c) in order to obtain a generalized and formal solution which can be applied to the interpretation of transient downhole temperature data.

By applying separation of variables to the homogeneous version of Eq. $(3 a-c)$ without the advective term, the following auxiliary problem is obtained:

$$
\nabla .\left(k_{T}(\boldsymbol{x}) \nabla \psi_{i}(\boldsymbol{x})\right)+\bar{\rho} \overline{\boldsymbol{C}_{p}} \sigma_{i}^{2} \psi_{i}(\boldsymbol{x})=0, \boldsymbol{x} \in V
$$

with homogeneous boundary conditions given by:

$$
a(\boldsymbol{x}) \psi_{i}(\boldsymbol{x})+b(\boldsymbol{x}) k_{T}(\boldsymbol{x}) \frac{\partial \psi_{i}(\boldsymbol{x})}{\partial \boldsymbol{n}}=0, \boldsymbol{x} \in S
$$

where $\psi_{i}(\boldsymbol{x})$ and $\sigma_{i}$ are the eigenfunctions and eigenvalues of the auxiliary problem, respectively. The norms of the auxiliary eigenvalue problem are given by $N_{i}=\int_{V} \bar{\rho} \overline{C_{p}} \psi_{i}^{2}(\boldsymbol{x}) \mathrm{d} V$. The appropriate inverse-transform pair is obtained at the onset of the problem and is given by:

$$
\begin{gathered}
\bar{T}_{i}(t)=\bar{\rho} \overline{C_{p}} \int_{V} \hat{\psi}_{i}(\boldsymbol{x}) T(\boldsymbol{x}, t) \mathrm{d} V \\
T(\boldsymbol{x}, t)=\sum_{i=1}^{\infty} \hat{\psi}_{i}(\boldsymbol{x}) \bar{T}_{i}(t)
\end{gathered}
$$

where $\hat{\psi}_{i}(\boldsymbol{x})$ is the normalized eigenfunction, given by $\hat{\psi}_{i}(\boldsymbol{x})=\psi_{i}(\boldsymbol{x}) / N_{i}^{1 / 2}$. Operating Eq. (3a) with $\int_{V} \hat{\psi}_{i}(\boldsymbol{x}) \mathrm{d} V$, one obtains:

$$
\begin{aligned}
& \frac{\mathrm{d} \bar{T}_{i}(t)}{\mathrm{d} t}+\rho_{f} C_{p f} \int_{V} \hat{\psi}_{i}(\boldsymbol{x})\left[\boldsymbol{v}(\boldsymbol{x}, t) . \nabla T_{(\boldsymbol{x}, t)}\right] \mathrm{d} V=-\sigma_{i}^{2} \bar{T}_{i}(t)+\bar{g}_{i}(t), \quad t>0, \\
& i=1,2, \ldots
\end{aligned}
$$

the integration of Eq. (7) can be done by substituting the inversion formula Eq. (6b) into Eq. (7):

$$
\frac{\mathrm{d} \bar{T}_{i}(t)}{\mathrm{d} t}+\sigma_{i}^{2} \bar{T}_{i}(t)+\sum_{j=1}^{\infty} A_{i j}^{*}(t) \bar{T}_{j}(t)=\bar{g}_{i}(t), \quad i=1,2, \ldots
$$


where

$$
A_{i j}^{*}(t)=\rho_{f} C_{p f} \int_{V} \hat{\psi}_{i}(\boldsymbol{x})\left[\boldsymbol{v}(\boldsymbol{x}, t) \cdot \nabla \hat{\psi}_{j}(\boldsymbol{x})\right] \mathrm{d} V
$$

and:

$$
\begin{aligned}
\bar{g}_{i}(t)= & \int_{V} \phi \beta T_{0} \frac{\partial p(\boldsymbol{x}, t)}{\partial t} \hat{\psi}_{i}(\boldsymbol{x}) \mathrm{d} V+\int_{V}\left(\phi c_{r}\left(p(\boldsymbol{x}, t)+\rho_{r} C_{p r} T_{0}\right)\right) \frac{\partial p(\boldsymbol{x}, t)}{\partial t} \hat{\psi}_{i}(\boldsymbol{x}) \mathrm{d} V \\
& +\int_{V}\left(\beta T_{0}-1\right) \boldsymbol{v}(\boldsymbol{x}, t) \cdot \nabla p_{(\boldsymbol{x}, t)} \hat{\psi}_{i}(\boldsymbol{x}) \mathrm{d} V+\int_{S} \frac{\varphi(\boldsymbol{x}, t)\left[\hat{\psi}_{i}(\boldsymbol{x})-k_{T}(\boldsymbol{x}) \frac{\partial \hat{\psi}_{i}(\boldsymbol{x})}{\partial \boldsymbol{n}}\right]}{a(\boldsymbol{x})+b(\boldsymbol{x})} \mathrm{d} S
\end{aligned}
$$

the transformed initial condition is:

$$
\bar{T}_{i}(t=0)=\bar{f}_{i}=\bar{\rho} \overline{C_{p}} \int_{V} \hat{\psi}_{i}(\boldsymbol{x}) f(\boldsymbol{x}) \mathrm{d} V, i=1,2 \ldots
$$

If analytical representation of the integrals involved in Eq. (8b-d) are not available, it will be necessary to numerically integrate these terms. This task, which was not necessary for the applications presented in this paper, can be performed by consolidated routines with controlled accuracy, such as the Gaussian quadrature.

Equation $(8 \mathrm{a}-\mathrm{d})$ form an infinite system of coupled ordinary differential equations for the transformed potentials. Computationally, the system of equations is truncated at the $N$ th row and column, with $N$ large enough to obtain the desired precision. In this work, the system of coupled ordinary differential equations is solved using the NDSolve routine of Mathematica Wolfram (2005). Other well established routines in packages of scientific computation, such as the IMSL Library, available in programming languages such as Fortran and Java could be used. After obtaining the transformed potentials, the inverse formula (Eq. 6b) can be used to obtain the desired potentials.

The formal solution presented in this section is a generalized and complete solution of the problem given by Eq. ( $3 \mathrm{a}-\mathrm{c})$ that can be applied to multi-dimensional problems in any orthogonal coordinate systems, with generalized boundary conditions that can be of the first, second and third kind. The initial condition can be chosen in order to represent the geothermal gradient or any other temperature distribution considered adequate to the problem being analyzed, as will be shown in the following applications.

\section{Applications}

Applications of the formal solution in the Cartesian coordinate system are now going to be presented. The applications consider a homogeneous reservoir, with constant porosity, permeability and rock thermal properties. It is important to note that the formal solution presented in "Formal Solution" section allows the incorporation of variations in rock thermal properties as well as permoporosities, by following the ideas presented in previous works (Naveira-Cotta et al. 2009; Deucher et al. 2016).

Figure 2 represents an illustration of the problem to be solved, with its boundary conditions and characteristic dimensions. 


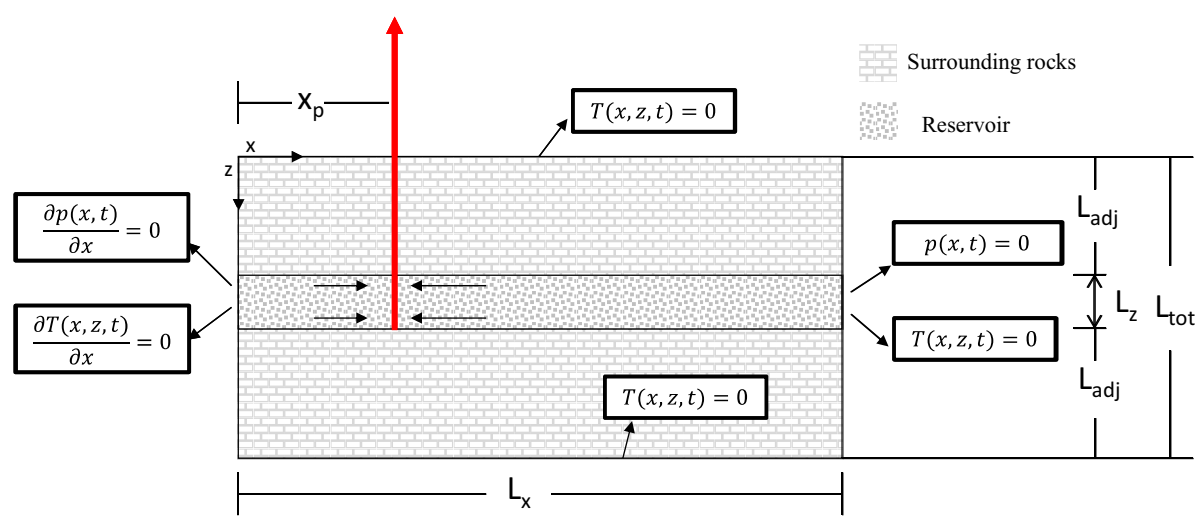

Fig. 2 Illustrative scheme of the applications

The problem in Fig. 2 consists of a two-dimensional heat transfer problem, where fluid flows toward a long horizontal well (placed at the position $x_{p}$ ) in a thin reservoir.

The modeling of the horizontal well as a plane sink assumes that:

(a) the streamlines can all be treated as linear in the reservoir and parallel to the $x$-axis. This is a common assumption in pressure transient analysis that is also applied to the transient temperature problem;

(b) the temperature, pressure and flow velocity fields are uniformly distributed in the $y$-direction, allowing the exclusion of the $y$-dimension from the analysis. This approximation holds if the well length is equal to the reservoir dimension parallel to the wellbore.

These assumptions were already used by Muradov and Davies (2011) to present analytical solutions for temperature interpretation in horizontal wells. It is important to keep in mind that these assumptions imply that the early-time radial and late-time radial flow regimes are not modeled in the following applications.

The modeling of the horizontal well as a plane sink also implies that convective and conductive heat transfer between the wellbore and the formation does not occur, as mentioned by App (2010), meaning that the sandface temperature is not affected by the flow inside the wellbore, i.e., the solution represents the pure reservoir response. If necessary, wellbore modeling techniques can describe the wellbore flowing temperature provided the pure reservoir response is known (Muradov and Davies 2008).

In order to validate the GITT solution, the results are going to be compared with the ones obtained with the asymptotic analytical solution presented by Muradov and Davies (2011) that will be considered as a reference solution. Aiming to preserve the conditions under which the reference solution is valid, the same reservoir parameters presented by Muradov and Davies (2011) are considered, as given in Table 1.

It is important to note that the rock compressibility in Table 1 is considered null due to the fact that the solution presented by Muradov and Davies (2011) did not consider the rock expansion/compression effects (2nd term on the right-hand side of Eq. 3a). This limitation is overcome by the GITT solution and the impacts of rock expansion/compression on the transient temperature behavior will be shown in "Two-dimensional Solution" section. 
Table 1 Data set for the applications presented in this work

\begin{tabular}{lll}
\hline Parameter & Metrical units & Field units \\
\hline Flowrate, $Q$ & $-0.04 \mathrm{~m}^{3} / \mathrm{s}$ & $-3456 \mathrm{~m}^{3} / \mathrm{d}$ \\
Permeability, $k$ & $10^{-14} \mathrm{~m}^{2}$ & $10.1 \mathrm{mD}$ \\
Porosity, $\phi$ & $30 \%$ & $30 \%$ \\
Total compressibility, $c_{t}$ & $10^{-9} \mathrm{~Pa}^{-1}$ & $10^{-4} \mathrm{bar}^{-1}$ \\
Viscosity, $\mu$ & $2 \times 10^{-4} \mathrm{Pas}$ & $0.2 \mathrm{cP}$ \\
Initial Pressure, $p_{0}$ & $3 \times 10^{7} \mathrm{~Pa}$ & $300 \mathrm{bar}$ \\
Reservoir thickness, $L_{z}$ & $2 \mathrm{~m}$ & $2 \mathrm{~m}$ \\
Length in $x$ direction, $L_{x}$ & $50 \mathrm{~m}$ & $50 \mathrm{~m}$ \\
Length in $y$ direction, $L_{y}$ & $2000 \mathrm{~m}$ & $2000 \mathrm{~m}$ \\
Well's position, $x_{p}$ & $0 \mathrm{~m}$ & $0 \mathrm{~m}$ \\
Thermal expansion coefficient, $\beta$ & $0.0008 \mathrm{~K}^{-1}$ & $0.0008^{\circ} \mathrm{C}^{-1}$ \\
Initial temperature, $T_{0}$ & $350 \mathrm{~K}$ & $76.85^{\circ} \mathrm{C}$ \\
Rock compressibility, $c_{r}$ & $0 \mathrm{~Pa}-1$ & $0 \mathrm{bar}-1$ \\
Thermal conductivity, $k_{T}$ & $2.148 \mathrm{~W} /(\mathrm{m} \mathrm{K})$ & $2.148 \mathrm{~W} /(\mathrm{m} \mathrm{K})$ \\
Fluid density, $\rho_{f}$ & $570 \mathrm{~kg} / \mathrm{m}^{3}$ & $570 \mathrm{~kg} / \mathrm{m}^{3}$ \\
Rock density, $\rho_{r}$ & $2200 \mathrm{~kg} / \mathrm{m}^{3}$ & $2200 \mathrm{~kg} / \mathrm{m}^{3}$ \\
Fluid-specific heat capacity, $C_{p f}$ & $1350 \mathrm{~J} /(\mathrm{kg} \mathrm{K})$ & $1350 \mathrm{~J} /(\mathrm{kg} \mathrm{K})$ \\
Rock-specific heat capacity, $C_{p r}$ & $1250 \mathrm{~J} /(\mathrm{kg} \mathrm{K})$ & $1250 \mathrm{~J} /(\mathrm{kg} \mathrm{K})$ \\
\hline
\end{tabular}

Applying the generalized solution of the HDE presented by Deucher et al. (2016) for a homogeneous reservoir, the following transient pressure behavior can be obtained:

$$
\begin{aligned}
p(x, t)= & p_{0}+\frac{8 L_{x}}{\pi^{2}} \frac{\mu Q}{k L_{y} L_{z}} \sum_{m=1}^{\infty} \frac{1}{(2 m-1)^{2}} \cos \left[\frac{(2 m-1) \pi}{2 L_{x}} x\right] \cos \left[\frac{(2 m-1) \pi}{2 L_{x}} x_{p}\right] \\
& \times\left[1-e^{-\eta \frac{(2 m-1)^{2} \pi^{2}}{4 L_{x}^{2}} t}\right]
\end{aligned}
$$

The time derivatives of the pressure can be obtained by direct differentiation of Eq. (9). The spatial derivatives were obtained by applying the integral balance to enhance the convergence of the eigenfunctions, as shown by Deucher et al. (2016), who also presented an analysis of the convergence of the pressure, as given by Eq. (9), as well as its spatial and time derivatives.

Given the parameters presented in Table 1 and the solution presented in Eq. (9), one can obtain the transient pressure behavior at the sandface, as shown in Fig. 3.

\subsection{One-dimensional Solution in the Cartesian Coordinate System}

The one-dimensional problem presented in this section does not consider heat transfer to the surrounding rocks (no $z$ direction considered in the problem of Fig. 2). This is representative of a hypothetic adiabatic reservoir. Applying the generalized formulation presented in Eq. $(3 a-c)$ to the one-dimensional problem in a homogeneous reservoir, one obtains: 


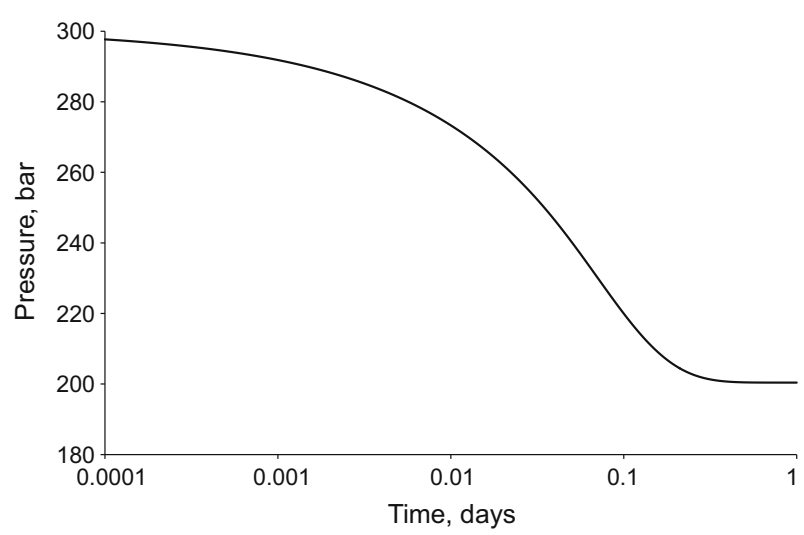

Fig. 3 Transient pressure behavior at the sandface $\left(x=x_{p}\right)$

Table 2 Convergence behavior of sandface temperature for different times

\begin{tabular}{|c|c|c|c|c|c|c|}
\hline \multirow[t]{2}{*}{ Time, days } & \multicolumn{6}{|c|}{$\begin{array}{l}\Delta T,{ }^{\circ} \mathrm{C} \\
\text { Number of terms }(N)\end{array}$} \\
\hline & 10 & 25 & 50 & 100 & 150 & 200 \\
\hline 0.1 & -0.29519 & -0.29959 & -0.30106 & -0.30174 & -0.30177 & -0.30178 \\
\hline 0.2 & -0.34680 & -0.35096 & -0.35246 & -0.35307 & -0.35311 & -0.35312 \\
\hline 0.5 & -0.34706 & -0.35041 & -0.35212 & -0.35254 & -0.35259 & -0.35260 \\
\hline 1 & -0.31866 & -0.32076 & -0.32274 & -0.32299 & -0.32304 & -0.32305 \\
\hline 5 & -0.08768 & -0.08446 & -0.08609 & -0.08640 & -0.08645 & -0.08646 \\
\hline 10 & 0.20747 & 0.21000 & 0.20913 & 0.20881 & 0.20877 & 0.20875 \\
\hline 20 & 0.80808 & 0.80066 & 0.79957 & 0.79925 & 0.79921 & 0.79919 \\
\hline
\end{tabular}

$$
\begin{aligned}
& \frac{\bar{\rho} \overline{C_{p}}}{k_{T}} \frac{\partial T(x, t)}{\partial t}+\frac{\rho_{f} C_{p f}}{k_{T}} \boldsymbol{v}(x, t) . \nabla T(x, t) \\
& =\frac{\phi \beta T_{0}}{k_{T}} \frac{\partial p(x, t)}{\partial t}+\frac{\left(p(x, t)+\rho_{r} C_{p r} T_{0}\right) \phi c_{r}}{k_{T}} \frac{\partial p(x, t)}{\partial t} \\
& \quad+\frac{\left(\beta T_{0}-1\right)}{k_{T}} \boldsymbol{v}(x, t) . \nabla p(x, t)+\nabla^{2} T(x, t), \\
& 0<x<L_{x}, \quad t<0 \\
& \quad \frac{\partial T(x, t)}{\partial x}=0, \quad x=0, \quad t>0 \\
& T(x, t)=0, \quad x=L_{x}, \quad t>0 \\
& T(x, t)=0, \quad 0<x<L_{x}, \quad t=0
\end{aligned}
$$

The mathematical manipulations involved in the application of the formal solution to problem given by Eq. (10a-d) are presented in Appendix 1.

Table 2 presents the convergence behavior of the sandface temperature $\left(x=x_{p}\right)$. The temperature sensors usually employed in oilfields have a maximum resolution of $0.001{ }^{\circ} \mathrm{C}$, thus, for practical purposes, the convergence of three decimal places is enough to consider the solution converged. 


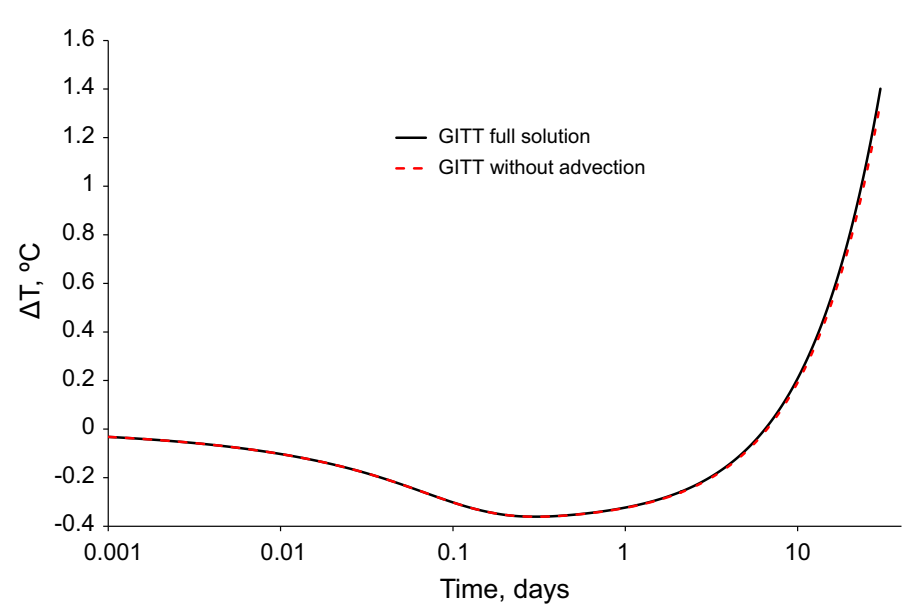

Fig. 4 Temperature variations at the sandface $\left(x=x_{p}\right)$ with and without the advective transport term

The analysis of Table 2 shows that convergence of three decimal places is obtained with 100 terms in the eigenfunction expansion for all the times analyzed and that 25 terms are enough to obtain the convergence of two decimal places, which is already enough for the analysis of temperature data collected by sensors of $0.01{ }^{\circ} \mathrm{C}$ resolution, such as fiber optic sensors.

The transient sandface $\left(x=x_{p}\right)$ temperature considering and neglecting advection is presented in Fig. 4, converged with 200 terms in the eigenfunction expansion.

As can be observed in Fig. 4, the advective transport term has a small impact on the sandface inflow temperature, at least for the parameters presented in Table 1 . The possibility of neglecting the advective term allows one to uncouple the transformed potentials $\left(A_{i j}^{*}(t)=0\right)$, making it possible to solve the transformed potentials analytically (not presented in this work) and/or with less computational effort. It is important to remember that the possibility of neglecting the advective term needs to be investigated case by case in order to avoid misinterpretations of the solutions.

\subsubsection{Impact of Initial Temperature Distribution}

Wellbore drilling and completion operations may disturb the original reservoir temperature. According to Kutasov (1999), temperature disturbance is affected by the duration of fluid circulation and shut-in periods, the circulating fluid temperature, the well radius and the thermal properties of the formations.

Aiming to evaluate the impact of these perturbations on the transient temperature behavior, the one-dimensional problem will be solved by considering a disturbed formation temperature in the near wellbore region given by:

$$
T(x, 0)=T_{1}+\frac{\left(T_{0}-T_{1}\right)}{e^{-\omega\left(x-x_{c}\right)}}, \quad 0<x<L_{x}, \quad t=0
$$

Considering Eq. (11) and the parameters presented in Table 3, the initial conditions that are evaluated in the following analysis are presented in Fig. 5.

The temperature disturbances presented in Fig. 5 do not aim to represent any specific situation. Indeed, the precise definition of temperature disturbance for a given wellbore at 
Table 3 Parameters of Eq. 11 to calculate different initial conditions

\begin{tabular}{llll}
\hline Initial condition (IC) & $T_{1}(\mathrm{~K})$ & $\omega\left(\mathrm{m}^{-1}\right)$ & $x_{c}(\mathrm{~m})$ \\
\hline IC1 & - & - & - \\
IC2 & -0.2 & 5 & 0.2 \\
IC3 & -0.4 & 5 & 0.5 \\
IC4 & -0.5 & 3 & 1.0 \\
IC5 & -0.5 & 2 & 2.0 \\
\hline
\end{tabular}

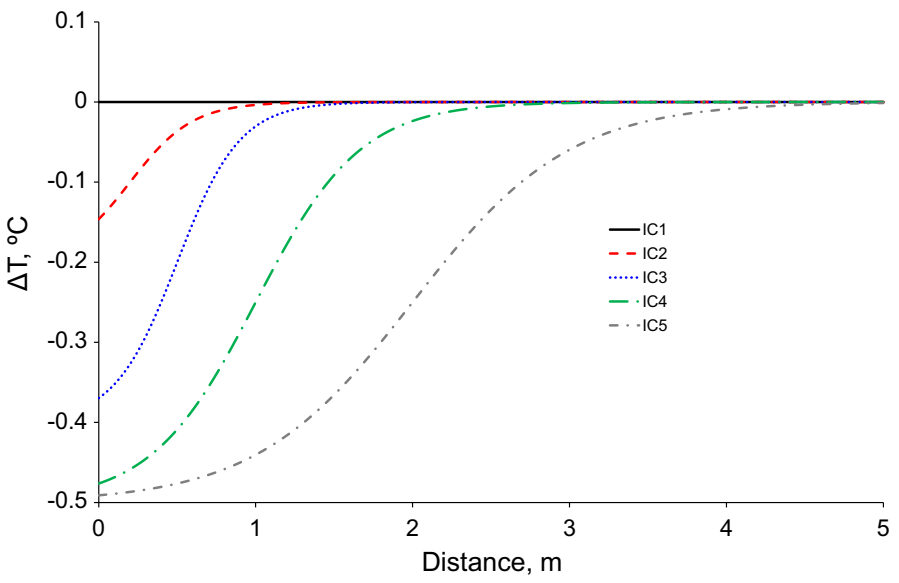

Fig. 5 Set of initial conditions

a given time is a difficult task because some of the factors affecting this disturbance are not readily available, such as the circulating fluid temperature, which varies with depth, the time and the circulating flowrate. In addition to that, the occurrence of fluid losses to high permeability layers poses even more obstacles in obtaining estimates of temperature disturbances in the near wellbore region. Thus, the initial conditions presented in Fig. 5 aim to represent uncertain and small temperature perturbations that could influence temperature transient analysis, especially during the first start up period of a well.

Solving the energy balance with the initial conditions represented by Eq. (11) with the parameters in Table 3, one obtains the impact of different initial conditions on the transient sandface $\left(x=x_{p}\right)$ temperature. The results presented in Fig. 6 are converged with 200 terms in the eigenfunction expansions.

Analyzing the results in Fig. 6, one can notice that small perturbations in the initial temperature (Fig. 5) have a significant effect on the transient thermal response, especially between 0.1 and 10 days, where both the values of the temperature and the shape of the curve (related to temperature derivatives) are affected by the initially disturbed temperature. For long times, the effects of the initial condition on the sandface temperature are very small, reducing to zero for sufficiently long times. The results presented in Fig. 6 show that perturbations of the initial reservoir temperature, if not adequately considered, could lead to errors in the interpretation of transient temperature data collected during the first start up period of a well, showing the importance of a proper cleanup of the well before transient temperature data interpretation. In addition to that, the analysis of transient temperature data 


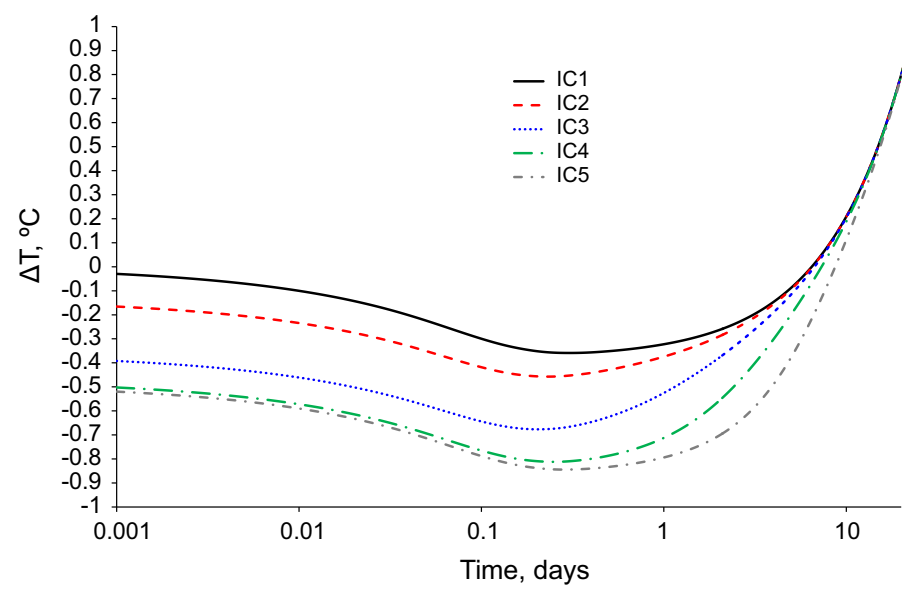

Fig. 6 Temperature variations at the sandface $\left(x=x_{p}\right)$ with different initial conditions

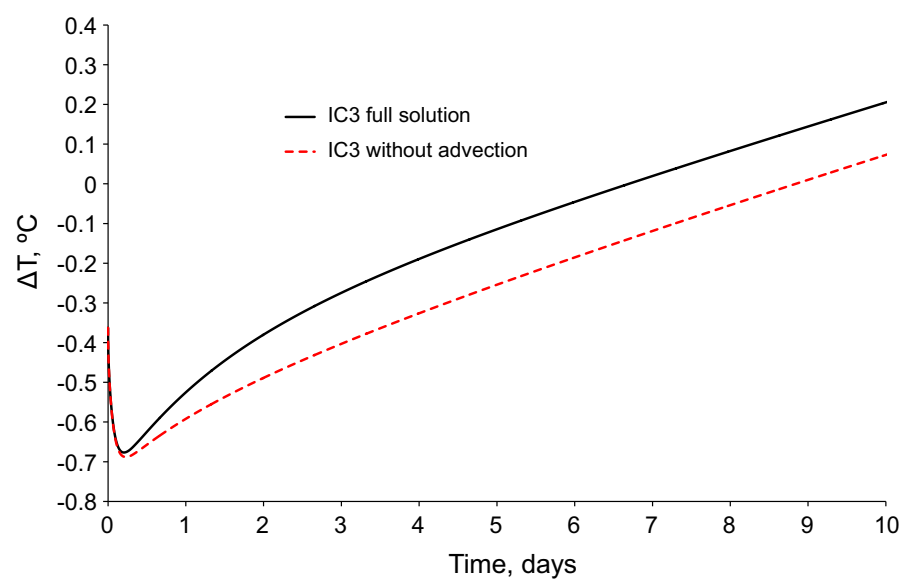

Fig. 7 Temperature variations at the sandface $\left(x=x_{p}\right)$ for IC3 with and without advection

can provide information of whether the near wellbore region is fully clean or not, as already mentioned by Theuveny et al. (2013).

The advective term, which represented a minor effect in the temperature calculations presented in Fig. 4, which considers uniform initial temperatures, has an important effect when the initial temperature distribution is not uniform. This can be observed in Fig. 7, which shows the transient sandface temperature with initial condition 3 (IC3) of Table 3, considering and neglecting the advective term.

It is important to remember that the results presented in this section did not consider the variations in the thermal properties of the fluids in the near wellbore region that are caused by drilling fluid invasion. However, even with this simplification, it is possible to conclude that minor temperature perturbations in the near wellbore region, which are generally present in the first start up period of a well, can have a significant impact on the transient thermal behavior and consequently on the interpretation of transient temperature data for reservoir characterization. 


\subsection{Two-dimensional Solution}

The two-dimensional problem presented in this section considers the heat transfer to the surrounding rocks, as depicted in Figs. 1 and 2. Applying the generalized formulation presented in Eq. (3a-c) to the two-dimensional problem in a homogeneous reservoir, one obtains:

$$
\begin{aligned}
& \frac{\bar{\rho} \overline{C_{p}}}{k_{T}} \frac{\partial T(x, z, t)}{\partial t}+\frac{\rho_{f} C_{p f}}{k_{T}} \boldsymbol{v}(x, z, t) \cdot \nabla T(x, z, t) \\
& =\frac{\phi \beta T_{0}}{k_{T}} \frac{\partial p(x, z, t)}{\partial t}+\frac{\left(p(x, z, t)+\rho_{r} C_{p r} T_{0}\right) \phi c_{r}}{k_{T}} \frac{\partial p(x, z, t)}{\partial t} \\
& \quad+\frac{\left(\beta T_{0}-1\right)}{k_{T}} \boldsymbol{v}(x, z, t) . \nabla p(x, z, t)+\nabla^{2} T(x, z, t), 0<x<L_{x}, \\
& 0<z<L_{\mathrm{tot}}, \quad t>0 \\
& \quad \frac{\partial T(x, z, t)}{\partial x}=0, \quad x=0, \quad t>0 \\
& T(x, z, t)=0, \quad x=L_{x}, \quad t>0 \\
& T(x, z, t)=0, \quad z=0, \quad t>0 \\
& T(x, z, t)=0, \quad z=L_{\mathrm{tot}}, \quad t>0 \\
& T(x, z, t)=0, \quad 0<x<L_{x}, \quad 0<z<L_{\mathrm{tot}}, \quad t=0
\end{aligned}
$$

where

$$
p(x, z, t)=\left\{\begin{array}{cl}
p(x, t), & L_{\mathrm{adj}}<z<L_{z}+L_{\mathrm{adj}} \\
0, & z \leq L_{\mathrm{adj}} \text { and } z \geq L_{z}+L_{\mathrm{adj}}
\end{array}\right.
$$

and the velocity vector, despite of being dependent of the $z$-direction, is not null only in the $x$-component, and is given by:

$$
\boldsymbol{v}(x, z, t)=\left\{\begin{array}{cl}
\boldsymbol{v}(x, t), & L_{\mathrm{adj}}<z<L_{z}+L_{\mathrm{adj}} \\
0, & z \leq L_{\mathrm{adj}} \text { and } z \geq L_{z}+L_{\mathrm{adj}}
\end{array}\right.
$$

It is important to mention that the initial condition (Eq. 12f) does not consider the geothermal heat flow, as this heat flow does not affect the results, according to the superposition principle, as already mentioned by Muradov (2010). The mathematical manipulations involved in the application of the formal solution to the problem given by Eq. (12a-f) are presented in Appendix 2.

Applying the same parameters presented in Table 1 and considering $L_{\text {adj }}=9 \mathrm{~m}$, which is long enough to guarantee the validity of boundary conditions $12 \mathrm{~d}-\mathrm{e}$ throughout the whole time domain for which the solution is desired (Deucher 2014), one obtains the results presented in Table 4, which represent the sandface temperature $\left(x=x_{p}\right)$ in a vertical $(z)$ position equivalent to the center of the reservoir. These results were obtained neglecting the advective term, due to its small impact on the solution, as shown in Fig. 4.

The analysis of Table 4 shows that 150 terms in each direction are sufficient to obtain the convergence of three decimal places. The computational time to solve the system of ordinary differential equations given by Eqs. (35-38) with 200 terms in each direction is 
Table 4 Convergence behavior of sandface temperature in the two-dimensional problem

\begin{tabular}{|c|c|c|c|c|c|c|}
\hline \multirow[t]{2}{*}{ Time, days } & \multicolumn{6}{|c|}{$\begin{array}{l}\Delta T,{ }^{\circ} \mathrm{C} \\
\text { Number of terms in each direction }(N)\end{array}$} \\
\hline & 10 & 25 & 50 & 100 & 150 & 200 \\
\hline 0.1 & -0.25698 & -0.33065 & -0.29779 & -0.30517 & -0.30195 & -0.30153 \\
\hline 0.2 & -0.30016 & -0.38438 & -0.34858 & -0.35389 & -0.35291 & -0.35281 \\
\hline 0.5 & -0.29580 & -0.37466 & -0.35147 & -0.35299 & -0.35305 & -0.35306 \\
\hline 1 & -0.26362 & -0.32592 & -0.31995 & -0.31969 & -0.31976 & -0.31976 \\
\hline 5 & -0.04113 & -0.01885 & -0.02628 & -0.02556 & -0.02562 & -0.02563 \\
\hline 10 & 0.17250 & 0.22470 & 0.21687 & 0.21760 & 0.21754 & 0.21753 \\
\hline 20 & 0.48541 & 0.55046 & 0.54246 & 0.54319 & 0.54313 & 0.54312 \\
\hline 40 & 0.91795 & 0.98859 & 0.98058 & 0.98130 & 0.98124 & 0.98123 \\
\hline
\end{tabular}

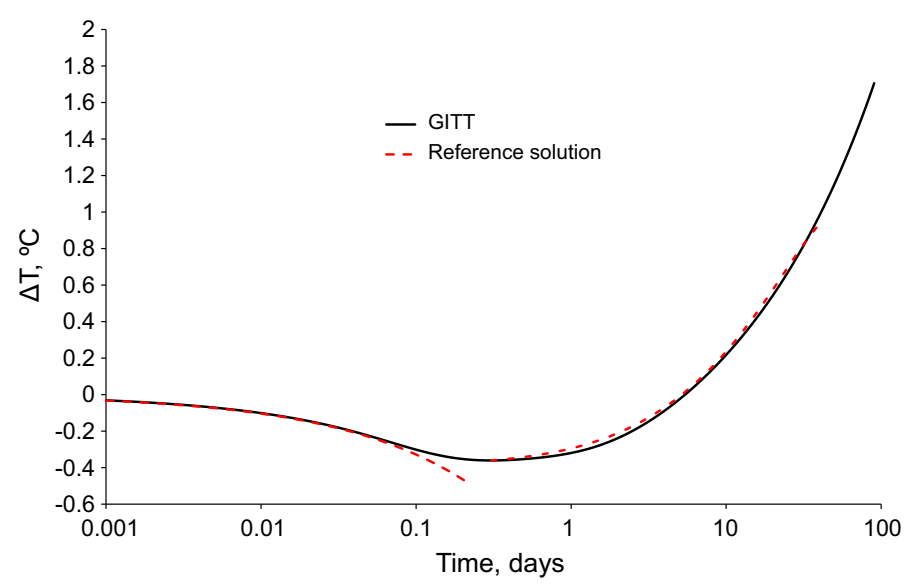

Fig. 8 Temperature variations at the wellbore $\left(x=x_{p}\right)$ given by the GITT and reference solution

3953 seconds, using an Intel $\mathbb{B}$ Core $^{\mathrm{TM}}$ i7-2960XM processor with 32MB RAM memory installed. Although not presented in this work, the convergence of the eigenfunction expansion for the two-dimensional problem can be accelerated, by properly reordering the terms in the eigenfunction expansions, as presented by Mikhailov and Cotta (1996).

Figure 8 shows the sandface temperature as obtained by the GITT with 200 terms in each direction of the eigenfunction expansion, as well as the results obtained by the asymptotic analytical solution presented by Muradov and Davies (2011), that is here considered as a reference solution for purposes of validation.

The analysis of Fig. 8 shows that the GITT and reference solutions are in good agreement. The deviations found between 0.1 and 0.2 days can be explained by the viscous dissipation effect that is not considered in the reference solution for short times. The reference solution is presented only for $t<40$ days, as it is not valid for longer times. The results presented in Fig. 8 validate the solution of the energy balance by the GITT presented in this work.

The GITT solution validated in Fig. 8 is obtained at a higher computational cost than the reference solution, but it brings important advances when compared to previous solutions: 
(a) It rigorously solves the energy balance using a single formulation valid for the whole space and time domains;

(b) It is continuous and differentiable;

(c) The association of the generalized solution of the energy balance with the generalized solution of the HDE presented by Deucher et al. (2016) allows one to solve the energy balance for the whole time domain, since the pressure solution considers all flow regimes (transient, late-transient and steady-state/pseudo-steady-state) in a single formulation;

(d) It is general and applicable to problems with variable flowrates and/or different boundary conditions, allowing, for example, to obtain the solution for bounded-reservoirs, where a steady-state flow behavior would not be achieved. These applications were not shown in this paper but can be found in Deucher (2014);

(e) It allows the consideration of several producing and thermally interacting layers, as shown ahead;

(f) It incorporates the effects of rock compressibility (2nd term on the right-hand side of Eq. 3a), as discussed next.

\subsubsection{Impact of Rock Compressibility}

When considering rock expansion/compression effects on the temperature behavior, one can assume that $p(x, z, t)+\rho_{r} C_{p r} T_{0} \cong p_{0}+\rho_{r} C_{p r} T_{0}$. The validity of this assumption can be checked by observing that $\rho_{r} C_{p r} T_{0} \gg p_{0}$. It is important to mention that this consideration is not mandatory for the application of the GITT solution to consider rock expansion/compression effects and can be alleviated in future works, if necessary.

Figure 9 shows an analysis of the sensitivity of the transient thermal behavior to variations in rock compressibility (the results shown consider 200 terms in each direction). Despite the effect of the rock compressibility on the total compressibility, to isolate the effect of the rock compressibility on the thermal behavior, the same total compressibility $\left(c_{t}=10^{-4} \mathrm{bar}^{-1}\right)$ was considered in all the cases presented in Fig. 9.

Figure 9 shows that the rock compressibility can significantly impact the transient temperature behavior, thus, rock compressibility may be a relevant parameter for downhole temperature modeling and interpretation. The results presented in Fig. 9 suggest that down-

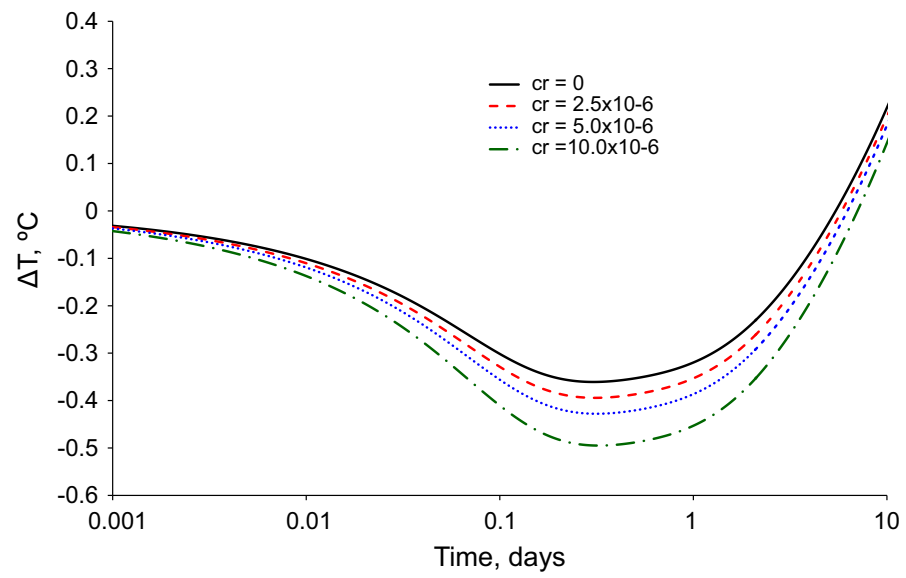

Fig. 9 Impact of rock compressibility on transient temperature behavior 
Fig. 10 Illustrative scheme of the conjugated heat transfer problem between two hydraulically disconnected reservoir layers

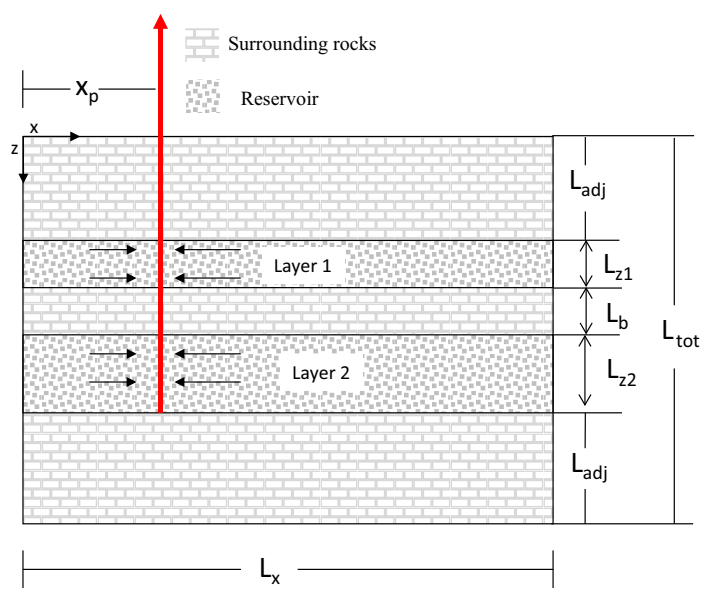

Table 5 Data set for Layer 2

\begin{tabular}{lll}
\hline Parameter & Metrical units & Field units \\
\hline Flowrate, $Q_{2}$ & $-0.011574 \mathrm{~m}^{3} / \mathrm{s}$ & $-1000 \mathrm{~m}^{3} / \mathrm{d}$ \\
Permeability, $k_{2}$ & $10^{-15} \mathrm{~m}^{2}$ & $1.01 \mathrm{mD}$ \\
Reservoir thickness, $L_{z 2}$ & $4 \mathrm{~m}$ & $4 \mathrm{~m}$ \\
Thickness of the flow barrier, $L_{b}$ & $2 \mathrm{~m}$ & $2 \mathrm{~m}$ \\
\hline
\end{tabular}

hole temperature have the possibility of being used to obtain in-situ estimates of the rock compressibility, although this application has yet to be proven in field data interpretation.

\subsubsection{Thermal Interaction Between Hydraulically Disconnected Reservoir Layers}

The solution presented in "Formal Solution" section can be used to solve the energy balance in a reservoir consisting of two (or more) hydraulically disconnected layers, such as represented in Fig. 10.

To solve this conjugated heat transfer problem, the energy balance is the same as given by Eq. 12a-f. The solution procedure is similar to the one presented in "Formal Solution" section, being it only necessary to calculate the source terms associated to pressure changes individually in each reservoir layer.

It is important to mention that for the top layer, the effects caused by a wellbore flowing fluid temperature different from the sandface temperature are not represented. As mentioned earlier, the proposed modeling implies that convective and conductive heat transfer between the wellbore and the formation does not occur, i.e., the solution represents the pure reservoir response. This same simplification was also applied by App and Yoshioka (2013) to model the transient sandface temperature in multilayered reservoirs.

The flow and reservoir properties considered in this example are the same used to obtain the results presented in Fig. 8, with the exception of the flowrate, permeability and reservoir thickness for Layer 2, which are presented in Table 5, along with the thickness of the impermeable layer separating Layers 1 and 2. 


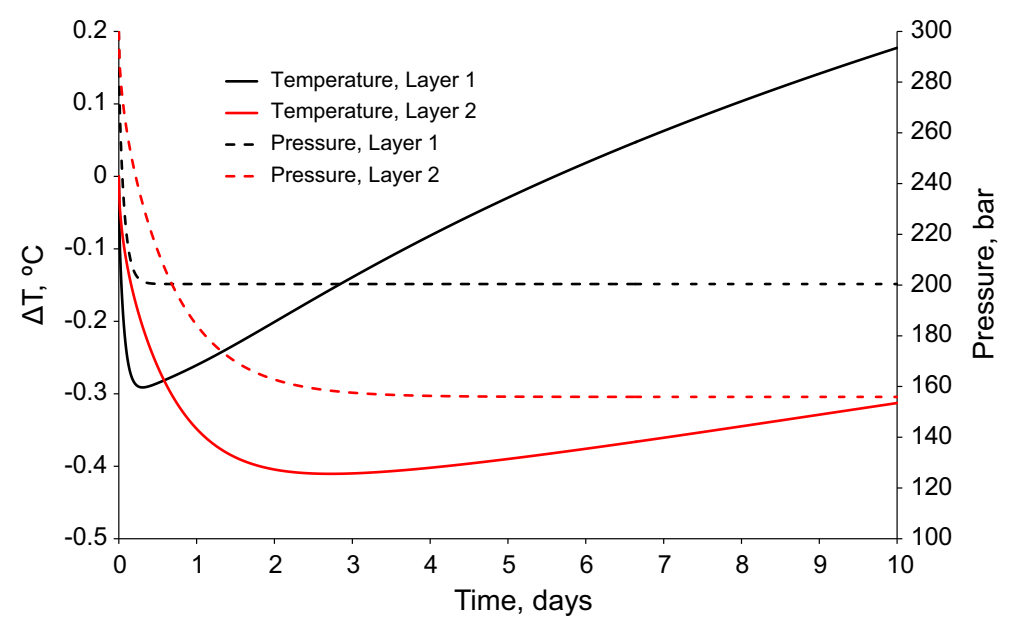

Fig. 11 Transient pressure and temperature behavior at $x=x_{p}$

$\Delta \mathrm{T},{ }^{\circ} \mathrm{C}$

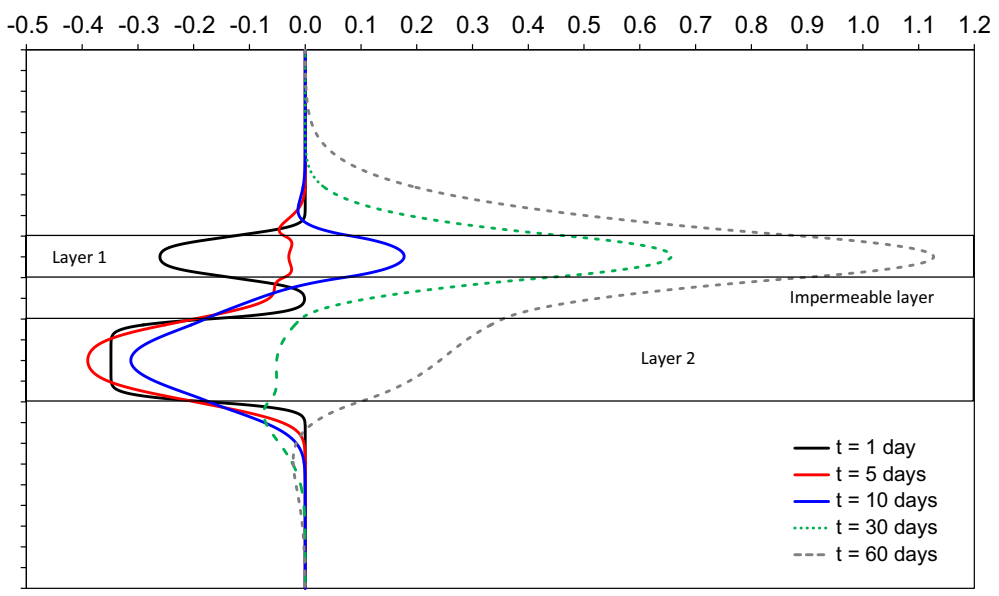

Fig. 12 Temperature at $x=x_{p}$ along the $z$ direction for different times

Figure 11 shows the transient pressure and temperature behavior at the well's position $\left(x=x_{p}\right)$ for the center of Layers 1 and 2 .

The analysis of Fig. 11 shows that after the initial period of temperature drop, Layer 1 temperature increase is more pronounced than the temperature increase in Layer 2. This can be explained by an analysis of the 3rd term on the right-hand side of Eq. (12a), which is higher in Layer 1 than in Layer 2, because of the higher flow velocities observed in Layer 1. However, the analysis of Fig. 11 does not make clear what are the effects caused by thermal interaction between Layers 1 and 2. These effects can be appreciated in Fig. 12, which shows the temperature variations at the position $x=x_{p}$ along the $z$ direction for different times. The positions of each layer as well as the position of the flow barrier are indicated in the plot.

The analysis of Fig 12 shows that for $t=1$ day, the temperature variations do not reach the center of the impermeable bed between reservoir Layers 1 and 2; up to this moment, 
the temperature in each layer behaves as if the other layer did not exist. For $t=5$ days, it is possible to observe variations in the temperature throughout the whole impermeable bed, meaning that the temperature in each layer is now dependent of the thermal behavior of the other layer. This becomes clear by observing the temperature behavior for $t=10$ days and beyond, where one perceives that the thermal interactions between Layers 1 and 2 cause temperature variations inside each layer that are not symmetric (i.e., the higher temperature variations do not happen in the center of the reservoir as would be expected for a single producing layer), especially for Layer 2, meaning that the thermal interaction between hydraulically disconnected layers may be important for transient temperature data interpretation under circumstances of practical interest, such as the interpretation of downhole temperature data in stratified reservoirs.

\section{Conclusions}

A hybrid solution of the energy balance in oil reservoirs was presented and validated. The solution is obtained by using the GITT, and the problem formulation considers thermal effects caused by the fluid and rock expansion/compression, as well as viscous dissipation. The application of the proposed solution follows a series of systematic steps, being general and applicable to multi-dimensional problems in any orthogonal coordinate system, with different kinds of boundary conditions.

The proposed solution brings about important advances for transient temperature modeling and interpretation: (a) it rigorously solves the energy balance for the whole space and time domains; (b) it is stable and computationally exact; thus, it can be used as a benchmark solution for purposes of validation; (c) it is continuous and differentiable, thus, more amenable to mathematical treatments for inverse problems and parameter estimation; (d) it solves the energy balance for all flow regimes (transient, late-transient and steady-state/pseudosteady-state) in a single formulation; (e) it allows the consideration of several producing and thermally interacting layers; (f) it incorporates the effects of rock compressibility.

In this paper, the generalized solution presented was applied to one- and two-dimensional problems in the Cartesian coordinate system, allowing for the interpretation of temperature data in situations where linear fluid flow occurs inside the reservoir (i.e., horizontal wells). The two-dimensional problem was tackled in a single domain formulation and allowed the model to consider heat transfer to the surrounding impermeable formations as well as an assessment of the impact of thermal interaction between two hydraulic disconnected reservoir layers on their transient temperature behavior.

Results obtained with the proposed solution show that: (a) small perturbations on the initial temperature can have a significant impact on transient temperature data interpretation, affecting the shape of the temperature curve, especially during the first start up period of a well; (b) thermal interaction between hydraulically disconnected reservoir layers, such as in stratified reservoirs, can cause significant impacts on the transient temperature behavior of the reservoir, pointing out the importance of considering these thermal interactions for appropriate downhole temperature data interpretation; (c) rock compressibility has significant impact on the transient temperature behavior and can be an important parameter for transient temperature modeling and interpretation.

Acknowledgements The first author thanks Petrobras for support in the development of this work. The second and third authors would like to acknowledge CNPq, CAPES and FAPERJ, research sponsoring agencies of 
the Brazilian and Rio de Janeiro State governments, for the continuous support of all their research activities over the years.

\section{Appendix 1}

Applying the method of separation of variables to the problem given by Eq. (10a-c), one can obtain the chosen eigenvalue problem:

$$
\begin{aligned}
& \frac{\partial^{2} \psi_{i}(x)}{\partial x^{2}}+\frac{\bar{\rho} \overline{C_{p}}}{k_{T}} \sigma_{i}^{2} \psi_{i}(x)=0,0<x<L_{x} \\
& \frac{\partial \psi_{i}(x)}{\partial x}=0, \quad \text { at } x=0 \\
& \psi_{i}(x)=0, \quad \text { at } x=L_{x}
\end{aligned}
$$

consulting the tabulated solutions in the textbook by Ozisik (1993), one can find the eigenfunctions, eigenvalues and norms of the eigenvalue problem:

$$
\begin{aligned}
\psi_{i}(x) & =\cos \left(\sqrt{\frac{\bar{\rho} \overline{C_{p}}}{k_{T}}} \sigma_{i} x\right) \\
\sigma_{i} & =\frac{(2 i-1) \pi}{2 L_{x}} \sqrt{\frac{k_{T}}{\bar{\rho} \overline{C_{p}}}} \\
N_{x, i} & =\frac{L_{x}}{2} \frac{\bar{\rho} \overline{C_{p}}}{k_{T}}
\end{aligned}
$$

using the generalized solution of "Formal Solution" section, the inverse-transform pair can be written as:

$$
\begin{aligned}
\bar{T}_{i}(t) & =\frac{\bar{\rho} \overline{C_{p}}}{k_{T}} \int_{0}^{L_{x}} \frac{1}{N_{x, i}^{1 / 2}} \cos \left[\frac{(2 i-1) \pi}{2 L_{x}} x\right] T(x, t) \mathrm{d} x \\
T(x, t) & =\sum_{i=1}^{\infty} \frac{1}{N_{x, i}^{1 / 2}} \cos \left[\frac{(2 i-1) \pi}{2 L_{x}} x\right] \bar{T}_{i}(t)
\end{aligned}
$$

performing the operations indicated in "Formal Solution" section, one obtains the coupled system of ordinary differential equations to be solved in order to obtain the transformed potentials.

$$
\frac{\mathrm{d} \bar{T}_{i}(t)}{\mathrm{d} t}+\left[\frac{(2 i-1) \pi}{2 L_{x}} \sqrt{\frac{k_{T}}{\bar{\rho} \overline{C_{p}}}}\right]^{2} \bar{T}_{i}(t)+\sum_{j=1}^{\infty} A_{i m}^{*}(t) \bar{T}_{m}(t)=\bar{g}_{i}(t), \quad i=1,2, \ldots
$$

where

$$
\begin{aligned}
A_{i m}^{*}(t)= & \frac{\rho_{f} C_{p f}}{k_{T} N_{x, i}^{1 / 2} N_{x, m}^{1 / 2}} \int_{0}^{L_{x}} \cos \left[\frac{(2 i-1) \pi}{2 L_{x}} x\right] \\
& \times \frac{d}{\mathrm{~d} x}\left\{\cos \left[\frac{(2 m-1) \pi}{2 L_{x}} x\right]\right\}\left(-\frac{k}{\mu} \frac{\partial p(x, t)}{\partial x}\right) \mathrm{d} x
\end{aligned}
$$




$$
\begin{aligned}
\bar{g}_{i}(t)= & \frac{1}{k_{T} N_{x, i}^{\frac{1}{2}}}\left\{\phi \beta T_{0} \int_{0}^{L_{x}} \cos \left[\frac{(2 i-1) \pi}{2 L_{x}} x\right] \frac{\partial p(x, t)}{\partial t} \mathrm{~d} x\right. \\
& +\phi c_{r} \int_{0}^{L_{x}}\left(p(x, t)+\rho_{r} C_{p r} T_{0}\right) \cos \left[\frac{(2 i-1) \pi}{2 L_{x}} x\right] \frac{\partial p(x, t)}{\partial t} \mathrm{~d} x \\
& \left.+\left(\beta T_{0}-1\right) \int_{0}^{L_{x}} \cos \left[\frac{(2 i-1) \pi}{2 L_{x}} x\right]\left[-\frac{k}{\mu}\left(\frac{\partial p(x, t)}{\partial x}\right)^{2}\right] \mathrm{d} x\right\}
\end{aligned}
$$

The transformed initial condition is given by:

$$
\bar{T}_{i}(0)=\bar{f}_{i}=0, \quad i=1,2 \ldots
$$

The calculation of the integrals in Eqs. 24 and 25 was performed using the platform Mathematica (Wolfram 2005), by substituting the expressions for $p(x, t)$ obtained from the solution of the HDE. It was possible to obtain all the integrals analytically for the cases presented in this paper, reducing the numerical task associated with the solution without any loss of accuracy.

\section{Appendix 2}

Applying the method of separation of variables to the problem given by Eq. (12a-f), one can obtain the chosen eigenvalue problems. The eigenvalue problem in the $x$-direction, as well as the eigenfunction and eigenvalues are the same given in Appendix 1; the $x$-direction norms in the two-dimensional problem are now given by $N_{x, i}=L_{x} / 2\left(\bar{\rho} \overline{C_{p}} / k_{T}\right)^{1 / 2}$. For the $z$-direction, we have:

$$
\begin{aligned}
& \frac{\partial^{2} \Omega_{j}(z)}{\partial z^{2}}+\frac{\bar{\rho} \overline{C_{p}}}{k_{T}} \chi_{j}^{2} \Omega_{j}(z)=0,0<z<L_{\text {tot }} \\
& \Omega_{j}(z)=0, \text { at } z=0 \\
& \Omega_{j}(z)=0, \text { at } z=L_{\text {tot }}
\end{aligned}
$$

Consulting the tabulated solutions in the textbook by Ozisik (1993), one can find the eigenfunctions, eigenvalues and norms of the eigenvalue problem in the $z$-direction:

$$
\begin{aligned}
\Omega_{j}(z) & =\sin \left(\sqrt{\frac{\bar{\rho} \overline{C_{p}}}{k_{T}}} \chi_{j} z\right) \\
\chi_{j} & =\frac{j \pi}{L_{\text {tot }}} \sqrt{\frac{k_{T}}{\bar{\rho} \overline{C_{p}}}} \\
N_{z, j} & =\frac{L_{\text {tot }}}{2}\left(\bar{\rho} \overline{C_{p}} / k_{T}\right)^{1 / 2}
\end{aligned}
$$


Using the generalized solution of "Formal Solution" section, the inverse-transform pair can be written as:

$$
\begin{array}{r}
\bar{T}_{i j}(t)=\frac{\bar{\rho} \overline{C_{p}}}{k_{T}} \int_{0}^{L_{x}} \int_{0}^{L_{\mathrm{tot}}} \frac{1}{N_{x, i}^{1 / 2} N_{z, j}^{1 / 2}} \cos \left[\frac{(2 i-1) \pi}{2 L_{x}} x\right] \sin \left(\frac{j \pi}{L_{\mathrm{tot}}} z\right) T(x, z, t) \mathrm{d} x \mathrm{~d} z \\
T(x, z, t)=\sum_{i=1}^{\infty} \sum_{j=1}^{\infty} \frac{1}{N_{x, i}^{1 / 2} N_{z, j}^{1 / 2}} \cos \left[\frac{(2 i-1) \pi}{2 L_{x}} x\right] \sin \left(\frac{j \pi}{L_{\mathrm{tot}}} z\right) \bar{T}_{i j}(t)
\end{array}
$$

Performing the operations indicated in "Formal Solution" section, one obtains the coupled system of ordinary differential equations to be solved in order to obtain the transformed potentials.

$$
\begin{aligned}
& \frac{d \bar{T}_{i j}(t)}{\mathrm{d} t}+\left\{\left[\frac{(2 i-1) \pi}{2 L_{x}} \sqrt{\frac{k_{T}}{\bar{\rho} \overline{C_{p}}}}\right]^{2}+\left[\frac{j \pi}{L_{\mathrm{tot}}} \sqrt{\frac{k_{T}}{\bar{\rho} \overline{C_{p}}}}\right]^{2}\right\} \bar{T}_{i j}(t) \\
& \quad+\sum_{m=1}^{\infty} \sum_{n=1}^{\infty} A_{i m j n}^{*}(t) \bar{T}_{m n}(t) \\
& =\bar{g}_{i j}(t), \quad i=1,2, \ldots, j=1,2, \ldots
\end{aligned}
$$

where

$$
\begin{aligned}
& A_{i m j n}^{*}(t)=\frac{\rho_{f} C_{p f}}{k_{T}} \frac{1}{N_{x, i}^{\frac{1}{2}} N_{z, j}^{\frac{1}{2}} N_{x, m}^{\frac{1}{2}} N_{z, n}^{\frac{1}{2}}} \int_{0}^{L_{x}} \int_{0}^{L_{\mathrm{tot}}} \cos \left[\frac{(2 i-1) \pi}{2 L_{x}} x\right] \\
& \times \frac{d}{\mathrm{~d} x}\left\{\cos \left[\frac{(2 m-1) \pi}{2 L_{x}} x\right]\right\} \sin \left(\frac{j \pi}{L_{\mathrm{tot}}} z\right) \frac{d}{\mathrm{~d} z}\left[\sin \left(\frac{n \pi}{L_{\mathrm{tot}}} z\right)\right] \\
& \times\left(-\frac{k}{\mu} \frac{\partial p(x, z, t)}{\partial x}\right) \mathrm{d} x \mathrm{~d} z \\
& \bar{g}_{i j}(t)=\frac{1}{k_{T} N_{x, i}^{1 / 2} N_{z, j}^{1 / 2}}\left\{\phi \beta T_{0} \int_{0}^{L_{x}} \int_{0}^{L_{\mathrm{tot}}} \cos \left[\frac{(2 i-1) \pi}{2 L_{x}} x\right] \sin \left(\frac{j \pi}{L_{\mathrm{tot}}} z\right) \frac{\partial p(x, z, t)}{\partial t} \mathrm{~d} x \mathrm{~d} z\right. \\
& +\phi c_{r} \int_{0}^{L_{x}} \int_{0}^{L_{\mathrm{tot}}}\left(p(x, z, t)+\rho_{r} C_{p r} T_{0}\right) \cos \left[\frac{(2 i-1) \pi}{2 L_{x}} x\right] \sin \left(\frac{j \pi}{L_{\mathrm{tot}}} z\right) \frac{\partial p(x, z, t)}{\partial t} \mathrm{~d} x \mathrm{~d} z \\
& \left.+\left(\beta T_{0}-1\right) \int_{0}^{L_{x}} \int_{0}^{L_{\mathrm{tot}}} \cos \left[\frac{(2 i-1) \pi}{2 L_{x}} x\right] \sin \left(\frac{j \pi}{L_{\mathrm{tot}}} z\right)\left[-\frac{k}{\mu}\left(\frac{\partial p(x, z, t)}{\partial x}\right)^{2}\right] \mathrm{d} x \mathrm{~d} z\right\}
\end{aligned}
$$

The transformed initial condition is given by:

$$
\bar{T}_{i j}(0)=\bar{f}_{i j}=0, i=1,2 \ldots, j=1,2 \ldots
$$

The solution of the problem given by Eqs. (35-38), which represent a specific case of generalized Eq. (8a-d), was obtained by substituting the expressions for the eigenfunction, eigenvalues and initial conditions into the generalized forms given in Eq. (8a-d). Further details on the procedure to derive Eq. (35) can be found in Cotta (1993).

The calculation of the integrals in equations $\mathrm{B} 10$ and $\mathrm{B} 11$ was performed using the platform Mathematica (Wolfram 2005), by substituting the expressions for $p(x, z, t)$ obtained from the solution of the HDE. It was possible to obtain all the integrals analytically for the cases presented in this paper, reducing the numerical task associated with the solution without any loss of accuracy. 


\section{References}

Almeida, A., Cotta, R.: Integral transform methodology for convection-diffusion problems in petroleum reservoir engineering. Int. J. Heat Mass Transf. 38, 3359-3367 (1995)

App, J.F.: Nonisothermal and productivity behavior of high-pressure reservoirs. SPE Journal 15, 50-63 (2010)

App, J.F., Yoshioka, K.: Impact of reservoir permeability on flowing sandface temperature: dimensionless analysis. SPE Journal 18, 685-694 (2013)

Carslaw, H., Jaeger, J.: Conduction of Heat in Solids. Clarendon Press, Oxford (1959)

Cotta, R.: Integral Transforms in Computational Heat and Fluid Flow. CRC Press, Boca Raton (1993)

Cotta, R.: Benchmark results in computational heat and fluid flow: the integral transform method. Int. J. Heat Mass Transf. 37, 381-393 (1994)

Deucher, R.H.: Solution of the energy balance in petroleum reservoirs by the generalized integral transform technique (in Portuguese) M.Sc. Thesis. Mechanical Engineering Program/COPPE, Federal University of Rio de Janeiro (2014)

Deucher, R.H., Couto, P., Bodstein, G.C.R.: Comprehensive solution for transient flow in heterogeneous porous media. Transp. Porous Media. 113(3), 549-566 (2016)

Duru, O.: Reservoir analysis and parameter estimation constrained to pressure, temperature and flowrate histories. Ph.D. Thesis, Dept. of Energy Resources Engineering, Stanford University (2011)

Hovanessian, S.: Pressure studies in bounded reservoirs. SPE J. 1, 223-228 (1961)

Kutasov, I.M.: Applied Geothermics for Petroleum Engineers. Elsevier, Amsterdam (1999)

Maubeuge, F., Didek, M.P., Deardsell, M.B., Caltagirone, J.P.: Temperature model for flow in porous media and wellbore. Presented at the SPWLA 35th Annual Logging Symposium, Tulsa, Oklahoma, USA, 19-22 June (1994)

Mikhailov, M., Cotta, R.: Ordering rules for double and triple eigenseries in the solution of multidimensional heat and fluid flow problems. Int Commun. Heat Mass Transf 23, 299-303 (1996)

Mikhailov, M., Ozisik, M.: Unified Analysis and Solutions of Heat and Mass Diffusion. Wiley, New York (1984)

Muradov, K.M.: Temperature modelling and real-time flow rate allocation in wells with advanced completion. Ph.D. Thesis, Institute of Petroleum Engineering, Heriot-Watt University (2010)

Muradov, K.M., Davies, D.R.: Prediction of temperature distribution in intelligent wells. Presented at the SPE Russian Oil and Gas Technical Conference and Exhibition, Moscow, Russia, 28-30 October (2008)

Muradov, K.M., Davies, D.R.: Novel analytical methods of temperature interpretation in horizontal wells. SPE J. 16(03), 637-647 (2011)

Naveira-Cotta, C.P., Cotta, R.M., Orlande, H.R.B., Fudym, O.: Eigenfunction expansions for transient diffusion in heterogeneous media. Int J Heat Mass Transf 52(21-22), 5029-5039 (2009)

Ozisik, M.: Heat Conduction. Wiley, New York (1993)

Rahman, N., Bentsen, R.: Use of an integral transform technique for comprehensive solutions to transient flow problems in homogeneous domains. s.1., s.n (2000)

Rahman, N., Bentsen, R.: Comprehensive Solutions for Transient-Flow Problems in 3D Homogeneous Domains. s.1., s.n (2001)

Ramanazov, A., Nagimov, V.: Analytical model for the calculation of temperature distribution in the oil reservoir during unsteady fluid flow. Oil Gas Bus. 1 (2007). (http://ogbus.ru/eng/authors/Ramazanov/ Ramazanov_2e.pdf)

Sui, W., Zhu, D., Hill, A.D., Ehlig-Economides, C.: Model for transient temperature and pressure behavior in commingled vertical wells. SPE Paper no. 115200-MS. Presented at the SPE Russian Oil and Gas Technical Conference and Exhibition, Moscow (2008)

Theuveny, B.C., Mikhailov, D., Spesivtsev, P., Starostin, A., Osiptsov, A.A., Sidorova, M., Shako, V.: Integrated approach to simulation of near-wellbore and wellbore cleanup. SPE Paper no. 166509-MS. Presented at the SPE Annual Technical Conference and Exhibition, New Orleans, Lousiana, USA (2013)

Wolfram, S.: The Mathematica Book, Version 5.2. Wolfram Media, Cambridge (2005)

Yoshioka, K., Hill, A.D., Dawkrajai, P., Lake, L.W.: A comprehensive model of temperature behavior in a horizontal well. SPE Paper no. 95656-MS. Presented at the SPE Annual Technical Conference and Exhibition, Dallas, Texas, USA, 9-12 October (2005) 